\title{
Emittance Measurement and Data Analysis for the SSC Linac Injector Lab
}

\author{
J.E. Hébert, P. Datte, F.W. Guy, N.C. Okay, K. Saadatmand, J. Sage, D.M. Wetherholt, W.A. Whittenberg \\ Superconducting Super Collider Laboratory" \\ 2550 Beckleymeade Avenue, Suite 125 \\ Dallas, TX 75237-3997
}

\section{Abstract}

The Superconducting Super Collider (SSC) ion source/LEBT produces and focuses a $35 \mathrm{keV} 30 \mathrm{~mA} \mathrm{H}^{-}$ beam into the RFQ. The beam emittance $\left(\varepsilon_{n-m e}\right)$, and its other characteristics, are measured using a slit and collector diagnostic system. The resultant data is analyzed by two separate applications. Both the hardware and software involved will be discussed. Result examples will be presented.

\section{Introduction}
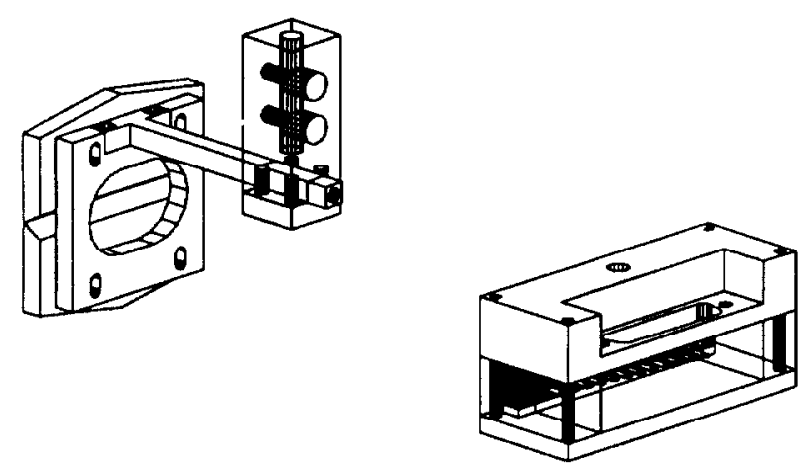

Figure 1 Downstream view of the SSC slit and collector

In the SSC Linac Injector Lab, a slit and collector diagnostic system is used to measure the transverse emittance of the $\mathrm{H}^{-}$beam at the output of the ion source, LEBT, and RFQ'. The beam conditions are quite diverse, depending on which system is being evaluated.

This paper will discuss the system hardware, the software used to control the hardware and make the measurements, and the software used to analyze the data. Finally, the performance of the system will be discussed with particular interest on certain techniques and algorithms which have significantly extended the limitations of the system.

\section{Hardware}

Slits

The slits used in this system were designed for use in a low energy $(35 \mathrm{keV})$, highly divergent beam. The knife edges have a flat specified as $0.001 " \pm 0.0005^{\prime \prime}$. This allows very narrow gaps with very high angular acceptance.

Note in figure 1 that the slit design allows for longitudinal positioning. Note also that the beveled sides of the knife edges are opposite the beam incident surface. This is practical since thermal loading is not a concern at the

- Operated by the University Research Association, Inc. for the U.S. Department of Energy, under contract No. DE-AC3589ER40486. intended beam energy. These features allow the incident edges of the slit (the plane of measurement) to be positioned directly in the plane of interest. This is of particular significance at low energies where emittance growth due to space charge is most significant.

A final feature of the slit design is its ability to serve as a faraday cup. The slits are electrically isolated from the vacuum vessel by an insulating bracket. The slits are biased in the normal way, and the output is measured on an oscilloscope. Comparisons of this system to the solid faraday cup used previously have shown no measurable difference. This feature eliminates the need for an additional device in the beamline, and the associated hardware risks.

\section{Collectors}

The collectors are 48 channel devices, and were built at Los Alamos National Laboratory. Each channel consists of an $0.008^{\prime \prime}$ foil of copper, laminated with a $0.002^{\prime \prime}$ layer of insulating film. The signal produced on each channel is preamplified and fed into an 11 bit ADC.

\section{Software}

\section{Measurement and Motion Control}

The application used to control the slit and collector motion, and measure the beam, is named Emit. It was written in-house using TACL (Thaumaturgic Automated Control Logic), which was developed at CEBAF.

During an emittance scan, the position of the slit (slit[i]) defines the $\mathrm{X}$-axis coordinate of phase space, while the position of the collector (coll[i]) defines the $X^{\prime}$-axis coordinate of each channel (or wire) of the collector. This gives rise $\omega$ a finite plane of phase space covered by any given system. The $\mathrm{X}$-axis limits are defined by the limits of travel of the slit. The limits of the $X^{\prime}$-axis, however, are

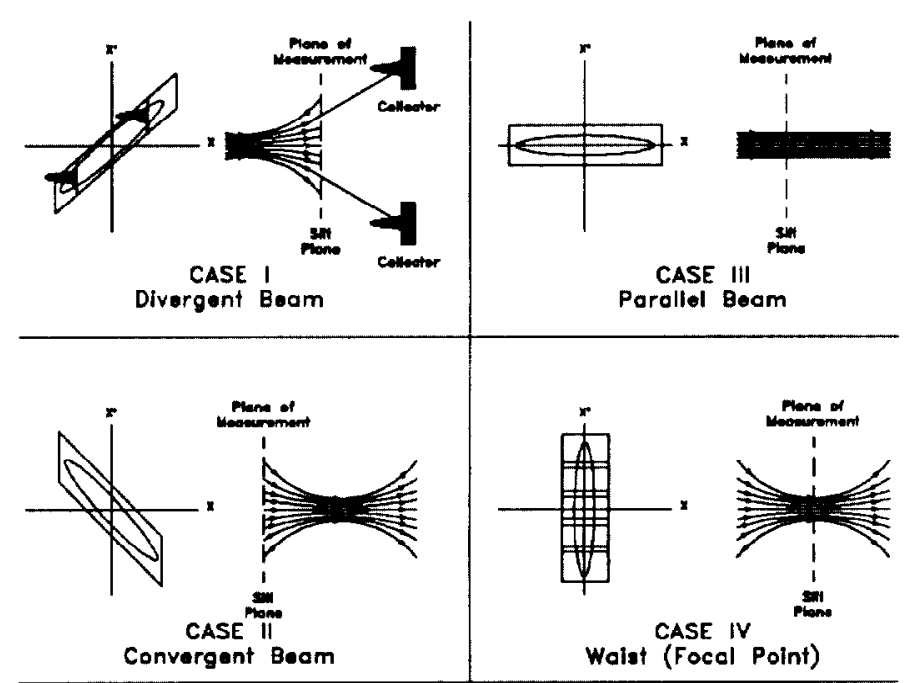

Figure 2 Examples of phase space ellipse orientations 
defined by the angular acceptance of the slit, or the limits of travel of the collector, whichever is least.

Figure 2 shows four possible phase space ellipse orientations. Each case requires a different motion of the collector, relative to the slit, to sweep out that region of the plane.

Consider case I, a divergent beam. When the slit is at the first edge of the beam, the angular distribution of the ellipse is at a positive angle. At this point, the collector must be at a more positive position than the slit. When the slit has passed through the beam to the opposite edge, the angular distribution is at a negative angle, with the collector at a more negative position than the slit.

For this reason, the collector must traverse a greater distance than the slit. The algorithm used in this system, similar to that used by Los Alamos National Laboratory ${ }^{2}$, defines the motion of the collector as a factor times the slit motion (or distance traversed). This factor is specified by the experimenter as the ratio of collector-to-slit motion, or simply the ratio.

In addition to the ratio (rat), the experimenter specifies a slit offset (s_off), a collector offset (c_off), a step size (step_sz), and the total number of steps (steps) the system is to make. From these parameters, the slit and collector motion is defined as follows:

$$
\begin{gathered}
\text { slit }[0]=s \text { soff }+(\text { step_sz*steps }) / 2 \\
\text { coll[ } \left.[0]=\mathrm{c} \_ \text {off }+ \text { rat*(step_sz*steps }\right) / 2
\end{gathered}
$$

For $\mathrm{i}=1$ through steps

$$
\begin{gathered}
\text { slit }[i]=\operatorname{slit}[i-1]+\text { step_sz } \\
\text { coll }[i]=\text { coll }[i-1]+\text { rat*step_sz }
\end{gathered}
$$

Notice that the motion of the slit and collector will sweep out a parallelogram in phase space centered about the slit and collector offsets. The ratio specified by the experimenter will determine the slope of the parallelogram. In case I the ratio would be positive. In case II, a convergent beam, the ratio would be negative. Case III would require a ratio of unity, and case IV is a special case which will be addressed later.

At each step, the system samples the beam for a time duration (gate), typically a few hundred nanoseconds, beginning at a specified time (delay). Both the gate duration and delay are specified by the experimenter. During this time, the signal from each channel will result in a digital count from 1 to 2048 in the associated ADC. These counts represent relative intensities which produce the angular distribution that makes up $\Delta \Theta$ (any vertical slice of the ellipse). The $X^{\prime}$ coordinate of each channel is calculated as follows:

$$
\begin{aligned}
& \text { For } \mathbf{j}=0 \text { through } 47 \\
& \text { wire[j] }= \\
& \operatorname{Tan}^{-1}\left(\left((\text { coll }[\mathrm{i}]-\operatorname{slit}[\mathrm{i}])+\left((24-\mathrm{i})^{*} \text { wire_sz }\right)\right) / \text { drift_ln }\right\}
\end{aligned}
$$

where wire_sz is the thickness of each channel, and drift_ln is the drift length between the slit and collector.

\section{Data Analysis}

The measured data is initially analyzed with Emit.
These analyses are printed as a color contour plot where the relative intensities are color coded through six (6) standard deviations. The associated numeric data are included on this plot.

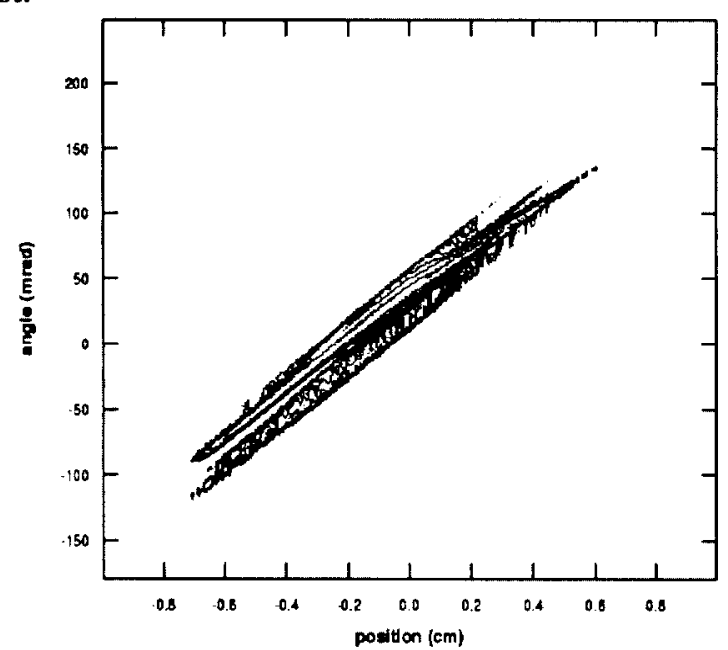

Figure 3 Plot of phase space data measured at the ion source (background subtracted).

The resultant data is then stored in a database from which it is extracted for further analysis off-line. This second analysis is performed with REANE analysis code, which was developed at, and provided by, Los Alamos National Laboratory. REANE provides the ability to plot the data as a set of six (6) contours corresponding to six (6) standard deviations, or as a set of percentiles of the total beam (see figure 3). The numeric data for REANE analyses are tabulated on a second printout.

Since background subtraction can't eliminate all extraneous signals from the data, REANE also allows the analyst to cut out the beam. Figure 3 shows an example of a raw data file after background subtraction, while figure 4 shows the same file after cutting.

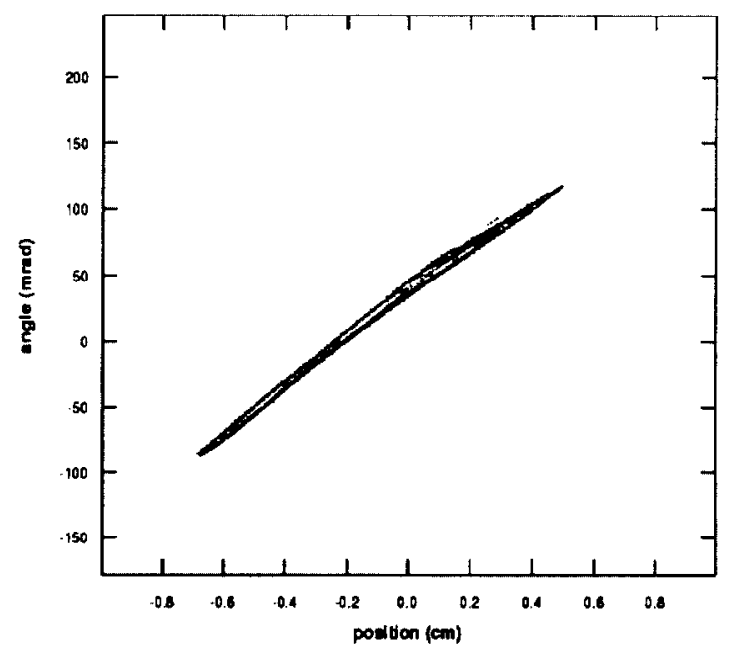

Figure 4 Plot of phase space data measured at the ion source (after cutting) 


\section{System Performance}

Ion Source Beam

Figures 3 and 4 are plots of a phase space measurement made at the ion source. In this case an extraneous cllipse appeared due to neutral particles. The collector channel amplifiers are biased negatively. As a result, secondary electrons generated by $\mathrm{H}^{\circ}$ particles produce a measurable signal which must be dealt with. A positive bias may eliminate the unwanted signals, but it would also adversely affect the resolution of the $\mathrm{H}^{-}$signals since recaptured electrons would not necessarily return to the channel of their origin.

As seen in figures 3 and 4 , this is not a significant problem in the vertical plane. The $\mathrm{H}^{-}$beam is deflected upwards slightly by the electron spectrometer. In the horizontal plane, however, both the $\mathrm{H}^{\circ}$ and $\mathrm{H}^{\circ}$ beams occupy the same phase space. To measure the horizontal axis of the ion source beam, a pair of electrostatic deflection plates were installed. With the plates grounded, the superimposed beams were measured. A second scan was made with $\pm 3.5 \mathrm{kV}$ on the deflection plates. The $\mathrm{H}^{0}$ particles were then measured, unperturbed by the $\mathbf{E}$ field, with all measurement parameters held constant. The $\mathrm{H}^{\circ}$ data was then subtracted from the superimposed data, leaving only the $\mathrm{H}^{-}$data as the difference. As a check, the deflected $\mathrm{H}^{-}$beam was also measured. The integrated sum of the separated $\mathrm{H}^{\circ}$ and $\mathrm{H}^{\circ}$ data was within $-5 \%$ of the superimposed beam.

\section{LEBT Beam}

Refer back to figure 2. Note that case IV shows the waist of the beam as the measurement plane. Since the area of the ellipse is the emittance, a conserved quantity, and is approximately

$$
\varepsilon \approx X_{\text {ewvelope }} X_{\text {eaveloge }}^{\prime}
$$

the $X^{\prime}$ envelope must increase as the beam diameter decrcases.

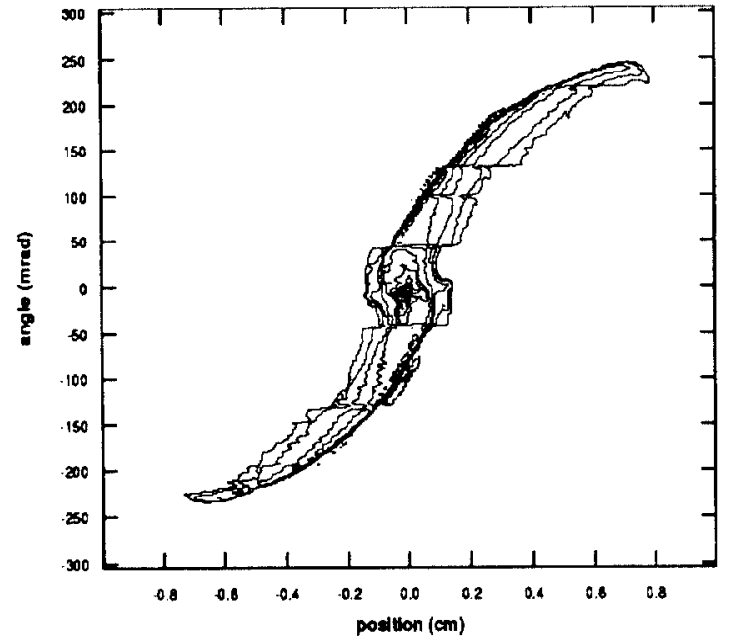

Figure 5 Plot of phase space data measured from the LEBT

Measuring the beam out of the LEBT was such a case. The focal point was the plane of interest, which is in fact a beam waist. The magnitude of the $X$ ' envelope made it impossible to capture the beam in a single scan. Instead, a series of scans, as many as seven (7), were required to capture the entire beam. Each scan was made with a ratio of collector to slit motion of unity, but at different collector offsets leaving only a small overlap between scans.

Analysis of the resultant data was accomplished via a program written to combine the separate scans into a single data file. This utility currently allows a single degree of freedom, the collector offset, requiring all other parameters be held constant from scan to scan. In the future, this utility will be modified to allow a second degree of freedom, the slit offset.

Figure 5 shows a typical phase space plot of the LEBT beam. Once the beam is cut with REANE analysis code, the seams become all but indiscernible.

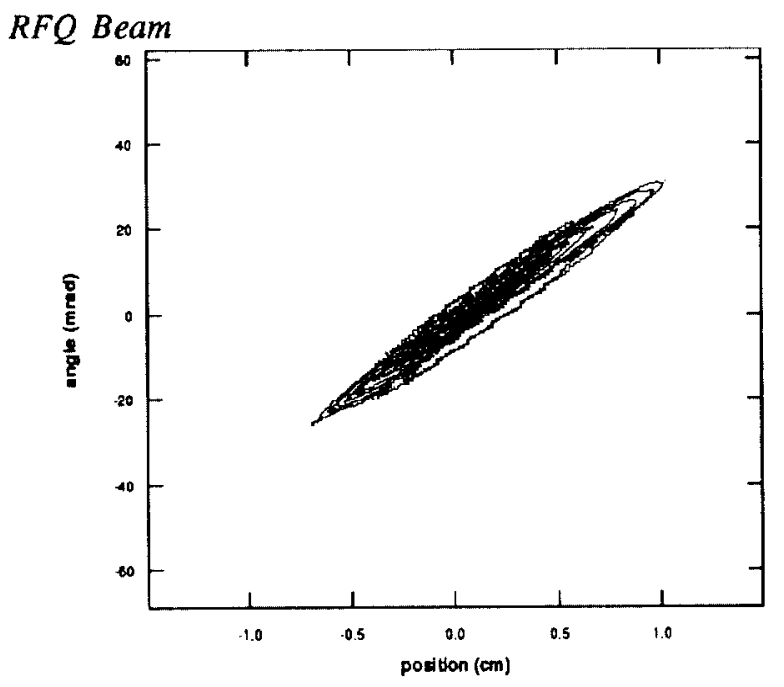

Figure 6 Sample emittance plot of beam out of RFQ

Measuring the RFQ beam posed a single challenge. The beam energy at the output of the RFQ is $2.5 \mathrm{MeV}$. To accommodate the thermal loading, new graphite plates for the slits were built. The knife edge flats were increased to $0.040^{\prime \prime}$ and the beveled faces were turned toward the beam. The rep-ratc of the beam was reduced from $10 \mathrm{~Hz}$ to $5 \mathrm{~Hz}$, and the current measuring capabilities of the slits were sacrificed in lieu of a thermally conductive path to the vacuum vessel.

Studies of the RFQ beam are ongoing, and the modified slit and collector system is performing exceptionally. Figure 6 is a typical emittance plot measured at the output of the RFQ.

\section{References}

Stanley Humphries, Jr., Charged Particle Beams, (Wiley, New York, 1990), p. $79-159$.

Saadatmand, K., (private communication). 